\title{
Topical amiodarone during cardiac surgery: Does epicardial application of amiodarone prevent postoperative atrial fibrillation?
}

\author{
David Greenstein, BM, Jordan Beau, BSc, Gary Gottlieb, PAC, Daniel Teller, PAC, and \\ Alexander Kulik, MD, MPH
}

\begin{abstract}
Objectives: Atrial fibrillation (AF) is a common complication after cardiac surgery. Topical amiodarone on the epicardium may help prevent postoperative $\mathrm{AF}$ while avoiding the side effects of its systemic administration. The purpose of this study was to evaluate the all-comer strategy of epicardial amiodarone application for the prevention of postoperative AF.
\end{abstract}

Methods: A retrospective observational study was performed that evaluated the incidence of new-onset AF in a consecutive series of cardiac surgery patients who were treated with either no amiodarone (historical control, $\mathrm{n}=100$ ), epicardial application of amiodarone mixed in a topical hydrogel $(n=50)$, or epicardial application of an amiodarone-soaked sealant patch $(\mathrm{n}=50)$. Perioperative data were compared between the 3 groups, with all patients receiving continuous postoperative telemetry to monitor for new-onset AF.

Results: The cohort consisted of 200 cardiac surgery patients (coronary bypass $82 \%$, valve surgery $24 \%$ ) who had no history of AF (mean age 71.0 years, $28 \%$ female). Among the 3 groups, the incidence of postoperative AF did not significantly differ, with 29 of $100(29 \%)$ patients in the historical control group having new AF, compared with 18 of $50(36 \%)$ in the amiodarone-hydrogel group, and 18 of $50(36 \%)$ in the amiodarone-patch group $(P=.56)$. The results did not differ when the analysis was restricted to coronary bypass patients only ( $\mathrm{n}=142,27 \%$ vs $38 \%$ vs $32 \%$, no-amiodarone vs amiodarone-hydrogel vs amiodarone-patch, respectively, $P=.56$ ). In multivariate logistic regression analysis, only older age $(P=.001)$ was significantly associated with new-onset $\mathrm{AF}$, but the use of topical amiodarone was not.

Conclusions: Routine epicardial application of topical amiodarone was not associated with a reduction in the incidence of new-onset postoperative $\mathrm{AF}$ in this observational study of older patients, leading us to question its role in contemporary cardiac surgical practice. (J Thorac Cardiovasc Surg 2017;154:886-92)

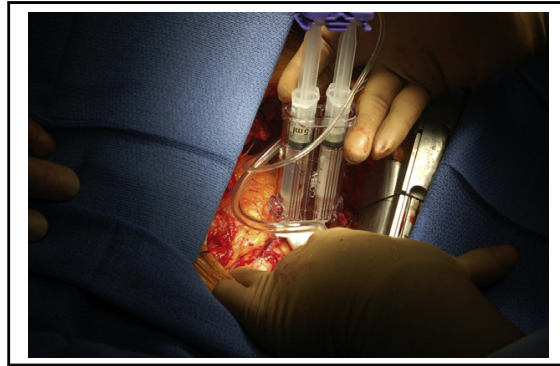

Epicardial application of amiodarone mixed in a topical hydrogel.

\section{Central Message}

Routine epicardial application of topical amiodarone was not associated with a reduction in the incidence of new-onset postoperative atrial fibrillation in this study.

\section{Perspective}

A recent trial suggested that topical amiodarone on the epicardium may help prevent postoperative atrial fibrillation after cardiac surgery. However, in this all-comers observational study of mostly older patients, routine topical amiodarone was not associated with a reduction in the incidence of new-onset postoperative atrial fibrillation, leading us to question its role in contemporary cardiac surgical practice.

See Editorial Commentary page 893.
Postoperative atrial fibrillation (AF) is a common and undesirable complication after cardiac surgery. Occurring in approximately $30 \%$ to $50 \%$ of patients, $1,2 \mathrm{AF}$ increases

From the Lynn Heart and Vascular Institute, Boca Raton Regional Hospital, and Charles E. Schmidt College of Medicine, Florida Atlantic University, Boca Raton, Fla.

Received for publication Oct 19, 2016; revisions received March 20, 2017; accepted for publication April 3, 2017; available ahead of print May 9, 2017.

Address for reprints: Alexander Kulik, MD, MPH, Lynn Heart and Vascular Institute,

Boca Raton Regional Hospital, 801 Meadows Rd, Suite 104, Boca Raton, FL

33486 (E-mail: alex_kulik@yahoo.com).

$0022-5223 / \$ 36.00$

Copyright (c) 2017 by The American Association for Thoracic Surgery

http://dx.doi.org/10.1016/j.jtcvs.2017.04.013 hospital length of stay and health care costs and is associated with a greater risk of postoperative heart failure, stroke, and mortality. ${ }^{2,3}$ Aside from routine perioperative beta-blockade, ${ }^{4}$ amiodarone is the most effective drug for the prevention and treatment of postoperative $\mathrm{AF}^{3,5}$

Scanning this QR code will take you to a supplemental video for the article. 


$$
\begin{aligned}
& \text { Abbreviations and Acronyms } \\
& \begin{aligned}
\mathrm{AF} & =\text { atrial fibrillation } \\
\mathrm{CABG} & =\text { coronary artery bypass graft } \\
\mathrm{CI} & =\text { confidence interval } \\
\mathrm{OR} & =\text { odds ratio }
\end{aligned}
\end{aligned}
$$

Amiodarone is a class III antiarrhythmic that also features some Class I, Class II, and antiadrenergic effects. By obstructing potassium channels, amiodarone decreases the membrane potential of myocardial cells and lengthens myocardial repolarization time. As a result, amiodarone reduces the incidence of postoperative $\mathrm{AF}$ by $50 \%$, in addition to reducing the risk of stroke, if administered orally or intravenously as prophylaxis in the preoperative period. ${ }^{6}$

Despite its excellent efficacy however, systemic administration of amiodarone has several disadvantages, including the requirement of a loading period and interactions with other drugs. ${ }^{3,7}$ Amiodarone contains high concentrations of iodine, which renders the drug highly lipophilic, leading to extensive accumulation in adipose tissue. Moreover, amiodarone is associated with many side effects, including nausea and vomiting, bradycardia, thyroid dysfunction, visual disturbances, pulmonary fibrosis, and liver toxicity. ${ }^{7,8}$ Ultimately, the challenging side effect profile of amiodarone has limited its routine and widespread use before cardiac surgery. ${ }^{9-11}$ Recent practice surveys have confirmed that, despite the weight of the evidence, most contemporary surgeons are reluctant to administer amiodarone as prophylaxis in the preoperative period. ${ }^{10,11}$

To avoid the side effects of systemic amiodarone, local drug-delivery techniques have been developed for the epicardial application of a slow, sustained-release of topical amiodarone. ${ }^{12-14}$ Direct diffusion of amiodarone into the target tissue would help minimize the disadvantages associated with its systemic administration. Promising results have been presented, including one recent pilot study in which the application of an amiodarone-releasing hydrogel on the epicardium reduced the incidence of postcardiac surgery AF from $26 \%$ to $8 \%(P<.01)$, with no safety concerns noted. ${ }^{14,15}$ After this trial's publication, ${ }^{14}$ we began to apply topical amiodarone routinely on the epicardium, with the hopes that the initially promising results also would be generalizable to our cardiac surgery practice. ${ }^{16,17}$ The purpose of the current study was to review our topical amiodarone experience using 2 alternative techniques and to evaluate its efficacy among all-comers for the prevention of new postoperative AF.

\section{METHODS}

\section{Study Design}

A retrospective observational study was performed focusing on 200 consecutive patients who underwent heart operations by one surgeon at a single center. All patients who underwent coronary artery bypass graft (CABG), valve, aortic, or combined surgery were included. Patients were specifically excluded if they had a history of previous AF. From December 2014 to October 2015, 50 consecutive patients received topical amiodarone via the hydrogel COSEAL technique. ${ }^{14}$ When doubts arose regarding its efficacy, ${ }^{16,17}$ we modified the topical technique and thereafter applied an amiodarone-soaked TachoSil patch to the epicardium for the next 50 patients from October 2015 to June 2016. The outcomes of these 2 amiodarone groups were then compared with a historical control group of 100 consecutive patients who had undergone surgery from November 2013 to December 2014, representing the era that immediately preceded the period of topical amiodarone application. Other than epicardial amiodarone, no other changes in surgical technique or postoperative care were initiated during the time period of this study.

Demographic and perioperative information was documented prospectively for each patient in a clinical database. Additional comorbidity data were collected from the hospital electronic medical records through retrospective chart review. The current study was approved by the Boca Raton Regional Hospital Research Committee (institutional review board) and Operating Room Committee (Boca Raton, Fla). The need for patient consent was waived because the safety and potential benefits of topical amiodarone already had been demonstrated in a published clinical trial. ${ }^{14}$

\section{Technique}

Patients in all 3 groups received similar intraoperative techniques, including the use of cardiopulmonary bypass, single crossclamp technique, and antegrade and retrograde blood cardioplegia. All patients received beta-blocker therapy in the morning before surgery, and beta blockers were resumed routinely on postoperative day 1 or 2 when hemodynamic stability was assured. No patient received prophylactic oral or intravenous amiodarone therapy before or after surgery.

The historical control group $(\mathrm{n}=100)$ received routine chest closure without amiodarone application. The pericardium was not reapproximated at the time of closure for any patient. Two mediastinal chest tubes were placed routinely anterior and inferior to the heart. Chest tubes were kept at $-20 \mathrm{~mm} \mathrm{Hg}$ suction and kept in position for 48 to 72 hours postoperatively.

For patients who were treated with amiodarone-hydrogel $(\mathrm{n}=50)$, $150 \mathrm{mg}$ of liquid amiodarone hydrochloride $(3 \mathrm{~mL})$ was added to a 2-mL COSEAL kit. COSEAL Surgical Sealant (Baxter Healthcare, Deerfield, Ill) is a sprayable polymeric matrix that forms a strong hydrogel that vigorously adheres to tissue. Each COSEAL kit includes 2 synthetic polyethylene glycol polymers that are mixed at application. Approximately $1.5 \mathrm{~mL}$ of amiodarone was added to each of the 2 syringes of the COSEAL kit after the polyethylene glycol powder had dissolved, yielding an amiodarone concentration of approximately $30 \mathrm{mg} / \mathrm{mL}$ in $5 \mathrm{~mL}$ of mixed solution. Shortly after preparation, the mixed amiodarone-hydrogel was applied before routine chest closure, via the previously described technique. ${ }^{14-16}$ Specifically, when an aerosolized applicator was used, the amiodarone hydrogel was sprayed diffusely over the epicardium of the right atrial lateral wall, the left atrial anterior wall, transverse sinus area, and the left atrial appendage when visible (Video 1).

For patients who were treated with an amiodarone-patch $(\mathrm{n}=50)$, a TachoSil Fibrin Sealant Patch $(4.8 \mathrm{~cm} \times 4.8 \mathrm{~cm})$ was immersed in $3 \mathrm{~mL}$ $(150 \mathrm{mg})$ of amiodarone hydrochloride liquid for longer than 5 minutes. The amiodarone-soaked TachoSil patch was then placed over the right atrial lateral wall and atrial appendage immediately before chest closure. TachoSil (Baxter Healthcare) is an absorbable sealant patch coated with fibrinogen and thrombin that often is used as an adjunct for hemostasis.

\section{Covariates}

Perioperative data were collected with a focus on surgical technique and patient comorbidities. For each patient, recorded preoperative 


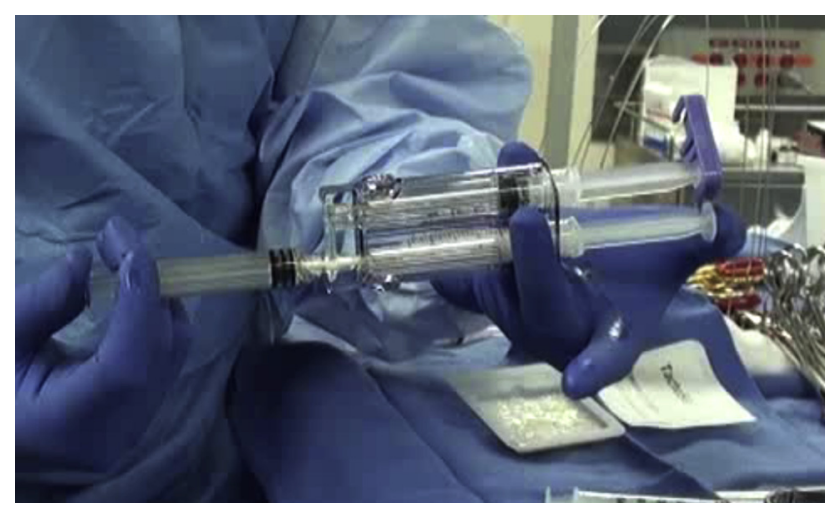

VIDEO 1. For patients treated with amiodarone-hydrogel, $150 \mathrm{mg}$ of liquid amiodarone hydrochloride $(3 \mathrm{~mL})$ is added to a $2 \mathrm{~mL}$ COSEAL kit (Baxter Healthcare). Approximately $1.5 \mathrm{~mL}$ of amiodarone is added to each of the 2 syringes of the COSEAL kit. The mixed amiodarone-hydrogel solution is then applied before chest closure via an aerosolized applicator. The amiodarone hydrogel is sprayed diffusely over the epicardium of the right atrial lateral wall, the left atrial anterior wall, transverse sinus area, and the left atrial appendage when visible. For patients treated with an amiodarone-patch, a TachoSil Fibrin Sealant Patch (Baxter Healthcare) is immersed in $3 \mathrm{~mL}(150 \mathrm{mg})$ of amiodarone hydrochloride liquid for longer than 5 minutes. The amiodarone-soaked TachoSil patch is then placed over the right atrial lateral wall and atrial appendage immediately before chest closure. Video available at: http:// www.jtcvsonline.org/article/S0022-5223(17)30700-6/addons.

characteristics included age, sex, body mass index, history of hypertension, hyperlipidemia, diabetes, previous percutaneous coronary intervention, preoperative creatinine level, previous myocardial infarction, recent acute coronary syndrome, previous transient ischemic attack or stroke, chronic obstructive pulmonary disease, peripheral vascular disease, and preoperative ejection fraction. Operative data included the surgical technique used and crossclamp and cardiopulmonary bypass times.

\section{Outcomes}

Postoperative data were recorded, including hospital length of stay and the occurrence of new postoperative AF within 30 days of surgery. Continuous telemetry was used to monitor for AF during the hospital stay. New postoperative AF was defined via the accepted Society of Thoracic Surgery definition: an episode of AF lasting more than 1 hour and/or requiring treatment (ie, administration of intravenous amiodarone, additional beta-blocker therapy, electrical cardioversion).

\section{Statistical Analysis}

Standard descriptive statistical analyses were used. Continuous data are presented as a mean \pm standard deviation, and categorical data are presented as proportions. Comparisons between the 3 study groups (no topical amiodarone vs amiodarone-hydrogel vs amiodarone-patch) were performed with analysis of variance, $\chi^{2}$ tests, or Fisher exact tests. Specifically, the 3 groups were compared in terms of the rate of new postoperative $\mathrm{AF}$ and postoperative complications. Additional sensitivity analysis was performed focused on patients who underwent CABG surgery only or any valve operation. Normality was determined with histogram evaluation and Kolmogorov-Smirnov tests. Because it lacked normal distribution, hospital length of stay was reported as a median with interquartile range and compared between the 3 groups with a KruskalWallis test.

Multivariate logistic regression analysis was then used to identify independent predictors of new postoperative AF for the entire cohort. All factors that differed between the 3 groups (Table 1) were considered in the model. Potential nonlinear associations between continuous variables and the outcome of postoperative AF also were evaluated. Factors tested in the model included the use of any topical amiodarone, the specific use of either amiodarone-hydrogel or amiodarone-patch (study group), age, sex, body mass index, medical comorbidities (listed in Table 1),

TABLE 1. Preoperative characteristics

\begin{tabular}{|c|c|c|c|c|}
\hline Characteristic & $\begin{array}{l}\text { No amiodarone } \\
\quad(\mathbf{n}=\mathbf{1 0 0})\end{array}$ & $\begin{array}{l}\text { Amiodarone-hydrogel } \\
\qquad(\mathbf{n}=\mathbf{5 0})\end{array}$ & $\begin{array}{l}\text { Amiodarone-patch } \\
\quad(\mathbf{n}=\mathbf{5 0})\end{array}$ & $\begin{array}{c}P \\
\text { value }\end{array}$ \\
\hline Time period & November 2013 to December 2014 & December 2014 to October 2015 & October 2015 to June 2016 & - \\
\hline Age, $y$ & $72.3 \pm 11.5$ & $69.9 \pm 13.5$ & $69.3 \pm 13.3$ & .31 \\
\hline Female sex & $25(25)$ & $15(30)$ & $15(30)$ & .73 \\
\hline Body mass index, $\mathrm{kg} / \mathrm{m}^{2}$ & $29.0 \pm 5.8$ & $28.8 \pm 6.1$ & $28.5 \pm 6.4$ & .88 \\
\hline Hypertension & $80(80)$ & $41(82)$ & $46(92)$ & .17 \\
\hline Hyperlipidemia & $90(90)$ & $41(82)$ & $42(84)$ & .34 \\
\hline Diabetes mellitus & $38(38)$ & $16(32)$ & $18(36)$ & .77 \\
\hline Preoperative creatinine level, $\mathrm{mg} / \mathrm{dL}$ & $1.24 \pm 0.69$ & $1.44 \pm 0.80$ & $1.11 \pm 0.49$ & .05 \\
\hline Previous transient ischemic attack or stroke & $9(9)$ & $2(4)$ & $1(2)$ & .22 \\
\hline Chronic obstructive pulmonary disease & $5(5)$ & $1(2)$ & $4(8)$ & .40 \\
\hline Peripheral vascular disease & $7(7)$ & $2(4)$ & $2(4)$ & .78 \\
\hline Previous percutaneous coronary intervention & $27(27)$ & $13(26)$ & $15(30)$ & .92 \\
\hline Previous (old) myocardial infarction & $15(15)$ & $7(14)$ & $4(8)$ & .55 \\
\hline Recent acute coronary syndrome & $29(29)$ & $15(30)$ & $13(26)$ & .94 \\
\hline Preoperative ejection fraction, $\%$ & $57.4 \pm 12.8$ & $53.5 \pm 12.6$ & $55.0 \pm 11.9$ & .18 \\
\hline CABG only & $70(70)$ & $32(64)$ & $40(80)$ & .19 \\
\hline Valve only & $15(15)$ & $6(12)$ & $7(14)$ & .96 \\
\hline Combined CABG and valve & $11(11)$ & $9(18)$ & $1(2)$ & .02 \\
\hline Cardiopulmonary bypass time, $\min$ & $140.2 \pm 48.2$ & $158.8 \pm 56.1$ & $147.7 \pm 48.1$ & .10 \\
\hline Crossclamp time, $\mathrm{min}$ & $102.8 \pm 36.3$ & $121.1 \pm 48.7$ & $109.2 \pm 38.1$ & .04 \\
\hline
\end{tabular}

Values are $\mathrm{n}(\%)$ or mean $\pm \mathrm{SD}$. $C A B G$, Coronary artery bypass graft. 
TABLE 2. Postoperative outcomes

\begin{tabular}{|c|c|c|c|c|}
\hline Outcome & $\begin{array}{c}\text { No amiodarone } \\
(\mathbf{n}=\mathbf{1 0 0})\end{array}$ & $\begin{array}{l}\text { Amiodarone-hydrogel } \\
(\mathbf{n}=\mathbf{5 0})\end{array}$ & $\begin{array}{l}\text { Amiodarone-patch } \\
(\mathbf{n}=\mathbf{5 0}) \\
\end{array}$ & $P$ value \\
\hline New postoperative atrial fibrillation & $29(29)$ & $18(36)$ & $18(36)$ & .56 \\
\hline Perioperative stroke & $1(1)$ & $2(4)$ & $1(2)$ & .56 \\
\hline Reopening & $1(1)$ & $2(4)$ & $2(4)$ & .29 \\
\hline New dialysis & $2(2)$ & $1(2)$ & $1(2)$ & 1.00 \\
\hline Postoperative death & $2(2)$ & $1(2)$ & $0(0)$ & .81 \\
\hline Postoperative length of stay, median days, interquartile range & $6,5-8$ & $7,5-10$ & $7,6-12$ & .06 \\
\hline
\end{tabular}

Values are $\mathrm{n}(\%)$ unless otherwise specified.

cardiopulmonary bypass time, crossclamp time, CABG surgery only, and any valve operation. Stepwise forward selection and backward elimination techniques were used, with $P=.20$ for entry and removal criteria, except for the use of topical amiodarone, which was forced into the model. Odds ratios (ORs) are reported with $95 \%$ confidence intervals (CIs). All reported $P$ values are 2-sided. All analyses were performed with Stata/MP version 11.2 (StataCorp, College Station, Tex).

\section{RESULTS}

\section{Patient Cohort}

The study cohort consisted of 200 patients who underwent cardiac surgery from 2013 to 2016. Complete data were available for the cohort. The mean age of the cohort was $71.0 \pm 12.5$ years, and $27.5 \%$ were female. Patients had a high incidence of preoperative comorbidities such as hypertension (84\%) and hyperlipidemia (86\%), and many patients had severe coronary disease as evident by a previous myocardial infarction $(13 \%)$ or recent acute coronary syndrome $(28 \%)$. No patient in the cohort had $\mathrm{AF}$ in the past. The majority of patients underwent $\mathrm{CABG}$ surgery $(82 \%)$, and a large percentage underwent valve surgery $(24 \%)$. The average hospital length of stay was $8.3 \pm 5.8$ days.

Table 1 describes the characteristics of 3 groups in the study. Patients in the amiodarone-hydrogel group had significantly greater preoperative creatinine levels, a greater rate of combined CABG-valve operations, and significantly longer crossclamp times (all $P<.05$ ), as compared with the amiodarone-patch and historical control (no-amiodarone) groups. These factors were all considered in the multivariate logistic model.

\section{Outcome Comparison}

Table 2 summarizes the clinical outcomes of the patient cohort. The incidence of new postoperative AF did not significantly differ between the 3 groups. The incidence of $\mathrm{AF}$ was $36 \%$ in both the amiodarone-hydrogel and amiodarone-patch groups, compared with $29 \%$ in the historical control (no-amiodarone) group $(P=.56)$. Among CABG patients only $(n=142)$, the rate of new postoperative AF also did not significantly different between the 3 groups $(27 \%$ vs $38 \%$ vs $32 \%$, no-amiodarone vs amiodarone-hydrogel vs amiodaronepatch, respectively, $P=.56$ ). When we focused on valve operations and excluded CABG-only patients $(\mathrm{n}=58)$, the rate of new postoperative $\mathrm{AF}$ also did not significantly different between the 3 groups $(33 \%$ vs $33 \%$ vs $50 \%$, no-amiodarone vs amiodarone-hydrogel vs amiodaronepatch, respectively, $P=.66$ ). The incidence of postoperative complications (stroke, reopening, new dialysis, and death) were similar between the 3 groups $(P=\mathrm{NS})$. There was a trend in favor of a longer hospital length of stay for the amiodarone-patch group $(P=.06)$.

\section{Multivariate Analysis}

Table 3 summarizes the results of the multivariate logistic regression analysis for new postoperative AF. Several variables reached or approached statistical significance on univariate analysis and were considered in the final multivariate model, including older age (OR, 1.1 per each additional year; $95 \% \mathrm{CI}, 1.0-1.1 ; P=.001)$ and a history of hypertension (OR, 2.5; 95\% CI, 1.0-6.3; $P=.06$ ). The use of any topical amiodarone $(P=.29)$ or the specific study group (no-amiodarone group vs amiodarone-hydrogel vs amiodarone-patch, $P=.34$ ) did not reach statistical significance on univariate testing. Preoperative creatinine level and crossclamp time also did not reach statistical significance on univariate testing. In the multivariate model, only older age (adjusted OR, 1.1 per each additional year; $95 \% \mathrm{CI}, 1.0-1.1 ; P=.001)$ was significantly associated with new AF. Neither the use of any topical amiodarone (adjusted OR, 1.5; 95\% CI, 0.8-2.9; $P=.19$ ) nor the specific study group (adjusted OR, 1.3; 95\% CI, 0.9-1.9; $P=.22$ ) were significantly associated with a reduction in the odds of $\mathrm{AF}$ when either variable was forced into the final model. Nearly identical multivariable results were generated when the analysis was isolated to $\mathrm{CABG}$ patients only.

\section{DISCUSSION}

$\mathrm{AF}$ is an important cause of patient morbidity and resource use after cardiac surgery. ${ }^{1-3}$ A potent antiarrhythmic, amiodarone can dramatically reduce the incidence of AF after heart operations if initiated as prophylaxis in the preoperative period. ${ }^{6}$ Despite these benefits, amiodarone is not instituted frequently as preventative therapy before surgery out of concerns for 
TABLE 3. Logistic regression analysis for new postoperative atrial fibrillation

\begin{tabular}{llc}
\hline \multicolumn{1}{c}{ Characteristic } & \multicolumn{1}{c}{ Univariate analysis } & Multivariate analysis \\
\hline Age (per additional year) & $\mathrm{OR}, 1.1 ; 95 \% \mathrm{CI}, 1.0-1.1 ; P=.001$ & Adjusted OR, $1.1 ; 95 \% \mathrm{CI}, 1.0-1.1 ; P=.001$ \\
Hypertension & $\mathrm{OR}, 2.5 ; 95 \% \mathrm{CI}, 1.0-6.3 ; P=.06$ & Adjusted OR, $2.2 ; 95 \% \mathrm{CI}, 0.8-6.0 ; P=.14$ \\
Valve surgery & $\mathrm{OR}, 1.4 ; 95 \% \mathrm{CI}, 0.7-2.8 ; P=.28$ & Adjusted OR, $1.7 ; 95 \% \mathrm{CI}, 0.8-3.5 ; P=.15$ \\
Any topical amiodarone & $\mathrm{OR}, 1.4 ; 95 \% \mathrm{CI}, 0.8-2.5 ; P=.29$ & Adjusted OR, $1.5 ; 95 \% \mathrm{CI}, 0.8-2.9 ; P=.19 *$ \\
Study group & $\mathrm{OR}, 1.2 ; 95 \% \mathrm{CI}, 0.8-1.7 ; P=.34$ & Adjusted OR, $1.3 ; 95 \% \mathrm{CI}, 0.9-1.9 ; P=.22 *$ \\
Diabetes mellitus & $\mathrm{OR}, 1.4 ; 95 \% \mathrm{CI}, 0.8-2.6 ; P=.26$ & - \\
Previous transient ischemic attack or stroke & $\mathrm{OR}, 0.4 ; 95 \% \mathrm{CI}, 0.1-1.9 ; P=.24$ & - \\
Cardiopulmonary bypass time (per additional minute) & $\mathrm{OR}, 1.0 ; 95 \% \mathrm{CI}, 1.0-1.0 ; P=.23$ & - \\
\hline
\end{tabular}

$O R$, Odds ratio; $C I$, confidence interval. *Both factors tested separately but not concurrently in the final model.

the serious side effects associated with its oral or intravenous administration, including lung and liver toxicity. ${ }^{7,9-11}$ However, if similar antiarrhythmic benefits could be achieved with its topical application, without the adverse consequences of its systemic administration, then epicardial amiodarone would prove to be an important and routine solution to the $\mathrm{AF}$ challenge that affects nearly $50 \%$ of postcardiac surgery patients.

With recent interest generated in the field, ${ }^{12-14}$ we sought to review our experience with topical amiodarone during cardiac surgery and to evaluate its efficacy for the prevention of new postoperative AF among all-comers. Although we were enthusiastic at first, especially given the promising data of an initial pilot trial, ${ }^{14-16}$ ultimately we were disappointed with the results. The rate of $\mathrm{AF}$ among 100 consecutive patients who served as historical controls $(29 \%)$ was no different compared with the rate of $\mathrm{AF}$ among the subsequent 50 patients $(36 \%)$ who were treated with the topical amiodarone-hydrogel (COSEAL). We subsequently modified the delivery technique using an amiodarone-soaked patch (TachoSil) placed on the epicardium. However, the rate of AF remained the same $(36 \%)$, and the incidence of $\mathrm{AF}$ was not significantly different amongst the 3 groups $(P=.56)$. When we isolated the analysis to CABG patients only, or when we controlled for other variables in a multivariate analysis, the use of topical amiodarone was not associated with a reduction in the odds of AF. As no benefit was seen in our 3-year experience, we have since discontinued its use in our cardiac surgery practice.

Topical amiodarone, either as an intrapericardial solution or a sustained-released system, was first evaluated in animal models, yielding encouraging early results. ${ }^{18,19}$ With the goals of reducing AF while avoiding the adverse effects of its systemic administration, Takeda and colleagues ${ }^{12}$ used a rabbit model to evaluate a biodegradable sustained-release amiodarone disc placed on the right atrium via sternal incision. The authors assessed tissue concentrations of amiodarone 3 days after implantation and noted successful local drug delivery and high amiodarone levels in right atrial tissue but minimal amiodarone concentrations in other adjacent organs
$(P<.01)$. Blood amiodarone concentrations, however, were below detectable levels. Importantly, compared with control animals, the investigators found that the rabbits treated with the amiodarone disc had an atrial effective refractory period that was significantly longer $(P=.03)$ and had a significantly greater $\mathrm{AF}$ threshold $(P<.01)$. Moreover, the amiodarone-treated rabbits had a significantly lower rate of $\mathrm{AF}$ when induced with rapid pacing $(P<.01){ }^{12}$

Thereafter, Bolderman and colleagues ${ }^{13}$ used a goat model to assess the efficacy of an amiodarone-releasing hydrogel applied to the right atrial epicardium. Goats treated with the hydrogel had amiodarone tissue concentrations in the right atrium that were significantly greater compared with other heart chambers or extracardiac tissues. Plasma amiodarone levels, in contrast, remained below the detection limit during the 28-day follow-up period. Moreover, goats that had received the amiodarone-hydrogel had significantly longer atrial effective refractory periods and conduction times and significantly reduced atrial responses to burst pacing $(P<.05)$ compared with the control animals. The authors concluded that, in their goat model, epicardial application of an amiodarone-releasing hydrogel led to successful local drug delivery and a reduction in vulnerability to atrial arrhythmias without the risk of extra-cardiac adverse side effects. ${ }^{13}$

Although these studies in animals generated provocative preliminary data, it remained uncertain whether the results from this translational research could be applied to the postcardiac surgery clinical setting. For example, the animals were free of coronary disease, and they were not subject to atrial cannulation sutures or the use of cardiopulmonary bypass. This raised doubts whether these models could accurately represent a typical cardiac surgery patient and the many factors that contribute to postoperative AF. Moreover, no chest tubes were used for the animals, and there was minimal bleeding to dilute or deplete the topically applied amiodarone, as would be expected to occur to some extent in humans. Finally, the investigators reported that topical amiodarone decreased $\mathrm{AF}$ that was induced with rapid pacing, but sustained AF did not occur in the animals. Clearly, the mechanisms of postcardiac surgery AF are 
unique from those of an animal model, where AF is induced with rapid-pacing.

Given the limitations of the studies in animals, it was with much interest that Feng and colleagues ${ }^{14}$ published the results of their pilot clinical trial, in which they evaluated the topical application of an amiodarone-releasing hydrogel during cardiac surgery. The authors randomized 100 patients to receive either usual care without the hydrogel $(\mathrm{n}=50)$ or amiodarone $1 \mathrm{mg} / \mathrm{kg}$ mixed with COSEAL (Baxter Healthcare) that was sprayed diffusely over the left and right atrial epicardium before sternal closure $(\mathrm{n}=50)$. A significant reduction in the incidence of postoperative AF was noted in the trial, with topical amiodarone patients having an AF rate of $8 \%$ compared with $26 \%$ for patients treated with usual care $(P<.01)$. Among the hydrogel-treated patients, amiodarone was measured in postoperative coronary sinus blood samples and in tissue biopsies from the right atrial wall, confirming successful local drug delivery. However, amiodarone was barely detectable in peripheral blood samples or extracardiac tissue biopsies after surgery. Of note, the authors reported no safety concerns associated with the amiodarone-hydrogel application in their trial. ${ }^{14}$ Although the authors performed an elegant study, it was not without its limitations. Their study population was relatively small, and no prestudy power calculations were provided. The design also was limited by the absence of blinding, a clear randomization process, and no placebo or sham therapy was used to serve as a control. ${ }^{16,17}$

Nevertheless, the question remains as to why the results achieved in our study differed so markedly from those seen by Feng and colleagues ${ }^{14,15}$ For the amiodaronehydrogel (COSEAL) patients, we applied the therapy using the same technique used by Feng and colleagues, ${ }^{14}$ except we used a larger amiodarone dose in our patient population for the purpose of simplicity $(150 \mathrm{mg}$ for all patients, instead of $1 \mathrm{mg} / \mathrm{kg}$ ). This, we expected, would have reduced the incidence of AF even further. We speculate that the lack of benefit seen in our experience relates to the differences in our practice and patient population compared with Feng and colleagues. ${ }^{14}$ First, all of our patients receive beta-blocker therapy before and after surgery, yet only $20 \%$ of patients were treated with beta blockers preoperatively in the trial of Feng and colleagues, ${ }^{14}$ and it is unclear whether beta blockers were administered in the postoperative period. ${ }^{14}$ Second, the mean age of patients included in our study was 71 years, compared with 52 years in the trial of Feng and colleagues, ${ }^{14}$ and our patients appeared to have more severe coronary disease, with a greater rate of previous myocardial infarction, and a higher prevalence of hypertension. $^{14}$

Moreover, Feng and colleagues ${ }^{14}$ routinely closed the pericardium in their operations, and they used an amiodarone powder that needed to be dissolved before application. On the contrary, we did not close the pericardium routinely, and we used liquid amiodarone in our study because amiodarone powder was not readily available at our hospital. Furthermore, in our study, we included patients who were undergoing either valve or coronary surgery, whereas only coronary patients were enrolled in the trial of Feng and colleagues. ${ }^{14}$ Although it was expected that the rate of AF would be greater in our cohort because it included valve patients, we still found no benefit associated with topical amiodarone even when we focused the analysis on coronary patients only. For the application of amiodaronehydrogel, we did not routinely dissect under the superior vena cava as described by Feng et al, ${ }^{14}$ but we did make every effort to spray the posterior left atrial wall and the atrial dome for each patient. These areas were particularly easier to reach during valve operations, yet no benefit was seen with topical amiodarone among the patients who underwent valve surgery in our analysis.

Finally, Feng and colleagues ${ }^{14}$ included patients in their trial who had a history of paroxysmal AF, some of whom underwent concurrent ablation surgery, ${ }^{14}$ whereas we specifically excluded patients with AF in our study. In our experience, nearly all patients with a history of preoperative $\mathrm{AF}$ will develop AF again after surgery, regardless of the use of topical amiodarone. Overall, we could not replicate the findings of Feng and colleagues ${ }^{14,15}$ in our cardiac surgery practice, putting the generalizability of their results in question. ${ }^{16,17}$ Perhaps the outcome of their pilot study was a chance finding. Ultimately, confirmation is needed in future large adequately powered clinical trials.

To our knowledge, the current analysis represents the largest study to date regarding the use of topical amiodarone for the prevention of postoperative AF after cardiac surgery. Nevertheless, the results of our study must be interpreted in the context of several limitations. First, the current paper is a retrospective review of prospectively collected data from a single surgeon and features a relatively modest sample size. However, power calculations suggested that we had approximately $96 \%$ power to detect a similar difference in the rate of AF to that reported by Feng and colleagues ${ }^{14}$ ( $26 \%$ vs $8 \%$ ), had that been present in our own experience, between patients who did or did not receive topical amiodarone (one-tail test, alpha 0.05). Since the present study was conducted at a single center, our results may not necessarily be generalizable to other cardiac practices with differing patient characteristics. Although it was not a prospective clinical trial, we believe the current analysis and comparison between the patient groups has important value since it is an "all-comers" study representing real-world experience, and no other changes in perioperative care were instituted during the time period of this study. We used multivariate analysis to adjust for differences in characteristics between the groups. Although we had considered applying propensity-score analysis to the 
study, in our experience, such adjustments nearly always yield identical results to those seen with conventional multivariate statistical tools.

Moreover, we did not specifically measure amiodarone concentrations in blood or tissue samples to confirm local drug delivery, because such testing already has been performed for the techniques used in this study by previous investigators. ${ }^{12-14}$ Finally, we did not perform sophisticated electrophysiology testing (such as conduction times or atrial responses to burst pacing), and instead we focused our study on clinically relevant outcomes, including the incidence of new AF. Ultimately, had a signal of AF reduction been present in our analysis, we had hoped to apply our data to subsequently power a prospective trial in the field. However, given the absent of benefit, we have since discontinued its use. Without sufficient evidence to support its routine application, we believe topical amiodarone therapy remains investigational at present.

In conclusion, routine epicardial application of topical amiodarone was not associated with a reduction in the incidence of new-onset postoperative $\mathrm{AF}$ in our observational study of older cardiac surgery patients. Until additional research is presented supporting its use, given its cost and the unclear benefits, we believe the routine use of topical amiodarone cannot be justified in contemporary cardiac surgical practice.

\section{Conflict of Interest Statement}

Authors have nothing to disclose with regard to commercial support.

\section{References}

1. Almassi GH, Schowalter T, Nicolosi AC, Aggarwal A, Moritz TE Henderson WG, et al. Atrial fibrillation after cardiac surgery: a major morbid event? Ann Surg. 1997;226:501-11; discussion 11-3.

2. Villareal RP, Hariharan R, Liu BC, Kar B, Lee VV, Elayda M, et al. Postoperative atrial fibrillation and mortality after coronary artery bypass surgery. J Am Coll Cardiol. 2004;43:742-8.

3. Camm AJ, Lip GY, De Caterina R, Savelieva I, Atar D, Hohnloser SH, et al. 2012 focused update of the ESC Guidelines for the management of atrial fibrillation: an update of the 2010 ESC Guidelines for the management of atrial fibrillation. Developed with the special contribution of the European Heart Rhythm Association. Eur Heart J. 2012;33:2719-47.
4. Kulik A, Ruel M, Jneid H, Ferguson TB, Hiratzka LF, Ikonomidis JS, et al. Secondary prevention after coronary artery bypass graft surgery: a scientific statement from the American Heart Association. Circulation. 2015;131: 927-64.

5. Wann LS, Curtis AB, January CT, Ellenbogen KA, Lowe JE, Estes NA III, et al. $2011 \mathrm{ACCF} / \mathrm{AHA} / \mathrm{HRS}$ focused update on the management of patients with atrial fibrillation (updating the 2006 guideline): a report of the American College of Cardiology Foundation/American Heart Association Task Force on Practice Guidelines. Circulation. 2011;123:104-23.

6. Bagshaw SM, Galbraith PD, Mitchell LB, Sauve R, Exner DV, Ghali WA Prophylactic amiodarone for prevention of atrial fibrillation after cardiac surgery: a meta-analysis. Ann Thorac Surg. 2006;82:1927-37.

7. Vassallo P, Trohman RG. Prescribing amiodarone: an evidence-based review of clinical indications. JAMA. 2007;298:1312-22.

8. Vorperian VR, Havighurst TC, Miller S, January CT. Adverse effects of low dose amiodarone: a meta-analysis. J Am Coll Cardiol. 1997;30:791-8.

9. Patel AA, White CM, Gillespie EL, Kluger J, Coleman CI. Safety of amiodarone in the prevention of postoperative atrial fibrillation: a meta-analysis. Am J Health Syst Pharm. 2006;63:829-37.

10. Price J, Tee R, Lam BK, Hendry P, Green MS, Rubens FD. Current use of prophylactic strategies for postoperative atrial fibrillation: a survey of Canadian cardiac surgeons. Ann Thorac Surg. 2009;88:106-10.

11. Lutz JM, Panchagnula U, Barker JM. Prophylaxis against atrial fibrillation after cardiac surgery: effective, but not routinely used-a survey of cardiothoracic units in the United kingdom. J Cardiothorac Vasc Anesth. 2011; 25:90-4.

12. Takeda T, Shimamoto T, Marui A, Saito N, Uehara K, Minakata K, et al. Topical application of a biodegradable disc with amiodarone for atrial fibrillation. Ann Thorac Surg. 2011;91:734-9.

13. Bolderman RW, Hermans JJ, Rademakers LM, de Jong MM, Bruin P, Dias AA, et al. Epicardial application of an amiodarone-releasing hydrogel to suppress atrial tachyarrhythmias. Int J Cardiol. 2011;149:341-6.

14. Feng XD, Wang XN, Yuan XH, Wang W. Effectiveness of biatrial epicardial application of amiodarone-releasing adhesive hydrogel to prevent postoperative atrial fibrillation. J Thorac Cardiovasc Surg. 2014;148:939-43.

15. Wang W, Mei YQ, Yuan XH, Feng XD. Clinical efficacy of epicardial application of drug-releasing hydrogels to prevent postoperative atrial fibrillation. J Thorac Cardiovasc Surg. 2016;151:80-5.

16. Beau J, Kulik A. Topical amiodarone to prevent postoperative atrial fibrillation: need for further study. J Thorac Cardiovasc Surg. 2016;151:600.

17. Wang W. Considerations for the success of topical amiodarone to prevent postoperative atrial fibrillation. J Thorac Cardiovasc Surg. 2016;151:600-1.

18. Darsinos JT, Karli JN, Samouilidou EC, Krumbholz B, Pistevos AC, Levis GM. Distribution of amiodarone in heart tissues following intrapericardial administration. Int J Clin Pharmacol Ther. 1999;37:301-6.

19. Bolderman RW, Hermans JJ, Rademakers LM, Jansen TS, Verheule S, van der Veen $\mathrm{FH}$, et al. Intrapericardial delivery of amiodarone and sotalol: atrial transmural drug distribution and electrophysiological effects. J Cardiovasc Pharmacol. 2009;54:355-63.

Key Words: amiodarone, atrial fibrillation, prophylaxis, cardiac surgery 